M.S. Sombrio,

https://orcid.org/0000-0001-8540-9347 H.M. Cardoso,

https://orcid.org/0000-0002-0636-218X

M.G. Luciani,

https://orcid.org/0000-0003-2567-4573

\section{Correlação entre os achados radiográficos e ecocardiográficos sugestivos de aumento cardíaco em cães: 104 casos}

[Correlation between radiographic and ecocardiographic findings suggestive of cardiomegaly in $104 \mathrm{dogs}]$

M.S. Sombrio ${ }^{1}$, H.M. Cardoso ${ }^{1}$, M.G. Luciani ${ }^{1}$, L.P. Souza ${ }^{1}$, P.E. Ferian ${ }^{2}$, J.A. Withoeft ${ }^{1}$, E. Zoboli ${ }^{1}$, T.R. Müller ${ }^{2}$
L.P. Souza,

https://orcid.org/0000-0001-7236-4434 P.E. Ferian,

https://orcid.org/0000-0003-2773-8008

J.A. Withoeft,

https://orcid.org/0000-0001-6246-2380 E. Zoboli,

https://orcid.org/0000-0002-0889-0170 T.R. Müller

https://orcid.org/0000-0002-7494-8588

\footnotetext{
${ }^{1}$ Aluno de pós-graduação - Universidade do Estado de Santa Catarina (UDESC) - Centro

de Ciências Agroveterinárias (CAV) - Lages, SC

${ }^{2}$ Universidade do Estado de Santa Catarina (UDESC) - Centro de Ciências

Agroveterinárias (CAV) - Lages, SC
}

\begin{abstract}
RESUMO
Radiografias torácicas e ecocardiogramas de 104 caninos foram avaliados e correlacionados quanto ao aumento das câmaras cardíacas. Os achados radiográficos foram correlacionados estatisticamente a fim de se estabelecer a acurácia do exame radiográfico na detecção do aumento cardíaco em comparação ao ecocardiográfico padrão-ouro não invasivo. A correlação entre os achados radiográficos indicativos de aumento cardíaco e os índices ecocardiográficos mostrou-se fraca, significativa somente para VHS versus relação átrio esquerdo/aorta $(\mathrm{r}=0,3136)$, eixo curto versus relação átrio esquerdo/aorta $(\mathrm{r}=0,3813)$ e eixo curto versus velocidade da onda $\mathrm{E}$ $(\mathrm{r}=0,2021)$. A acurácia da radiografia na determinação subjetiva de aumento das câmaras cardíacas foi razoável, variando entre $72,1 \%$ e $80,8 \%$. Em contrapartida, o VHS apresentou baixa acurácia $(50,9 \%)$ na detecção de cardiomegalia.
\end{abstract}

Palavras-chave: cão, radiografia, ecocardiograma, cardiomegalia, cardiopatia

\title{
ABSTRACT
}

Thoracic radiographs and echocardiograms of 104 canines were evaluated and correlated regarding cardiac chambers enlargement. The radiographic findings were statistically correlated in order to establish the accuracy of the radiographic examination in the detection of cardiac enlargement in comparison with the echocardiogram - non-invasive gold standard. The correlation between the radiographic findings indicative of cardiac enlargement and echocardiographic indexes was weak, significant only for VHS versus left atrium to aorta ratio $(r=0.3136)$, short axis versus left atrium to aorta ratio $(r=0,3813)$ and short axis versus $E$ wave velocity $(r=0.2021)$. The radiographic accuracy in the subjective determination of cardiac chamber enlargement was reasonable, ranging from $72.1 \%$ to $80.8 \%$. On the other hand, VHS presented low accuracy $(50.9 \%)$ in the detection of cardiomegaly.

Keyword: dog, radiography, echocardiogram, cardiomegaly, cardiopathy

\section{INTRODUÇÃO}

A radiografia torácica é utilizada rotineiramente como parte central do diagnóstico de cardiopatia, bem como no acompanhamento terapêutico em cães cardiopatas (Lamb e Boswood, 2002). O método tem evidente importância em casos de insuficiência cardíaca congestiva, tornando possível a visibilização de modificações associadas ao edema pulmonar cardiogênico, à efusão pleural e a alteração dos vasos pulmonares, além de permitir o diagnóstico de

Recebido em 9 de março de 2018

Aceito em 9 de outubro de 2018

E-mail: marinasiso@hotmail.com possíveis comorbidades torácicas não cardíacas, como a broncomalácia, o colapso de traqueia e a bronquite crônica (Lamb et at., 2001; Ferasin et al., 2013).

Entretanto, em pacientes que não estão em insuficiência cardíaca congestiva, o foco da avaliação passa a ser a morfologia cardiovascular, que se mostra um método pouco preciso por permitir somente análise subjetiva da silhueta cardíaca (Nakamaya et al., 2001), já que diversos fatores podem interferir na aparência radiográfica normal. Entre eles, cita-se a ampla variedade de conformações torácicas, 
especialmente inter-racial (Buchanan e Bücheler, 1995), o escore corporal, a idade, as fases dos ciclos respiratório e cardíaco em que a imagem foi obtida (Olive et al., 2015), o posicionamento radiográfico (Ruehl e Thrall, 1981) e a variação na interpretação interobservadores (Hansson et al., 2009).

Ainda, o tempo de desenvolvimento da doença cardíaca e seus efeitos sobre o miocárdio são determinantes quanto à ocorrência de sinais radiográficos (Lamb e Boswood, 2002), sendo esperada a cardiomegalia em casos de dilatação ou hipertrofia excêntrica do coração, e a alteração da forma como resultado de aumento em uma ou mais câmaras cardíacas. Lamb et al. (2001) detectaram maior acurácia da radiografia na detecção de anormalidades que impõem sobrecarga de volume ao coração, visto que culminam em hipertrofia excêntrica ou dilatação cardíaca, em relação àquelas que levam à sobrecarga de pressão - com consequente hipertrofia concêntrica.

Devido à subjetividade da radiografia na avaliação cardíaca, preconiza-se a ecocardiografia como modalidade padrão para avaliação de aumento cardíaco e anormalidades morfofuncionais, sendo um método dinâmico e não invasivo que permite diagnóstico mais acurado (Kienle e Thomas, 2005). O exame ecocardiográfico em modo bidimensional (modo B) permite analisar qualitativamente estruturas cardíacas e visibilizar em tempo real o seu funcionamento. $\mathrm{O}$ modo tempo-movimento (modo $\mathrm{M}$ ) fornece informação sobre a função cardíaca mediante o cálculo de múltiplos índices. Já a avaliação da hemodinâmica cardíaca é realizada por meio do modo Doppler (Armstrong et al., 2005).

Diante disso, o presente estudo tem como objetivo investigar a correlação existente entre os achados radiográficos e ecocardiográficos sugestivos de aumento cardíaco em cães, a fim de estabelecer a sensibilidade, a especificidade e a acurácia da radiografia torácica na determinação de cardiomegalia.

\section{MATERIAL E MÉTODOS}

Esta pesquisa foi aprovada pela Comissão de Ética no Uso de Animais, protocolada sob o Ceua, número 1238140316. Os pacientes selecionados para o estudo foram cães atendidos no Hospital de Clínica Veterinária "Lauro Ribas Zimmer" (CAV/UDESC) ou em uma clínica particular de Lages/SC, durante os anos de 2013 a 2016, sendo utilizados como critérios de inclusão: realização de exame de radiografia torácica em projeções ortogonais (laterolateral direita e ventrodorsal) e de ecocardiografia.

As análises radiográficas foram realizadas pelo mesmo avaliador - cego ao histórico clínico e aos resultados do ecocardiograma dos pacientes. Diversos parâmetros indicadores de aumento das câmaras cardíacas foram avaliados quanto a sua presença ou ausência. Em relação ao átrio esquerdo, os achados foram ainda graduados de forma subjetiva de 0 a 3 , sendo 0 sem indicativo de aumento; 1 , aumento discreto; 2 , moderado; e 3 , grave (Tab. 1).

Também foi calculado o vertebral heart size (VHS) na projeção lateral direita, composto pelos eixos curto e longo da silhueta cardíaca em relação à unidade de vértebras ocupadas a partir da quarta torácica. $\mathrm{Na}$ determinação de cardiomegalia, os parâmetros estabelecidos para as diferentes raças foram utilizados - incluindo Poodle (Pinto e Iwasaki, 2004), Boxer (Lamb et al., 2001), Dachshund, Shih Tzu, Lhasa Apso e York Shire (Jepsen-Grant et al., 2013) - para fins de interpretação radiográfica através do VHS. As demais raças ou pacientes sem raça definida foram classificados de acordo com o parâmetro VHS, determinado por Buchanan e Bücheler (1995).

Os exames ecocardiográficos foram realizados com transdutor de varredura setorial multifrequencial de $2-5 \mathrm{~Hz}$ (HD15 Ultrasound System Philips ${ }^{\circledR}$ Healthcare). As avaliações foram obtidas por meio dos modos bidimensional (B), unidimensional (M), Doppler pulsado (PW) e contínuo $(\mathrm{CW})$ e por mapeamento de fluxo em cores (CFM) e Doppler tecidual, sendo os dados e as imagens armazenados na memória do aparelho para posterior mensuração e cálculos. Esses exames foram realizados pelo mesmo avaliador - cego ao histórico clínico, ao exame físico e às radiografias. A obtenção das imagens e as avaliações ecocardiográficas foram realizadas conforme recomendado por Thomas et al. (1993) e Boon (2011). 
Tabela 1. Parâmetros radiográficos subjetivos analisados para determinação de aumento da silhueta cardíaca em cães, classificados de acordo com o escore de gravidade da alteração em: 0-ausente, 1discreta, 2-moderada, 3-severa

\begin{tabular}{|c|c|}
\hline Avaliação & Sinais radiográficos \\
\hline \multirow{7}{*}{$\begin{array}{l}\text { Cardiomegalia } \\
\text { generalizada }\end{array}$} & 1. Silhueta cardíaca de contorno arredondado. \\
\hline & $\begin{array}{l}\text { 2. Impressão de aumento do diâmetro apicobasilar e craniocaudal da silhueta cardíaca } \\
\text { pelo observador. }\end{array}$ \\
\hline & $\begin{array}{l}\text { 3. Deslocamento dorsal da traqueia intratorácica e tronco brônquico principal. } \\
\text { 4. Comparação com o número de espaços intercostais ocupados pelo coração } \\
{[\text { Ref.<3,5]. }}\end{array}$ \\
\hline & 5. Mensuração pelo VHS (vertebral heart size) - eixo curto + eixo longo. \\
\hline & $\begin{array}{l}\text { 6. Redução da distância entre a silhueta cardíaca e o diafragma, podendo haver } \\
\text { sobreposição de ambos. }\end{array}$ \\
\hline & $\begin{array}{l}\text { 7. Largura da silhueta cardíaca ultrapassando } 2 / 3 \text { da largura da cavidade torácica em } \\
\text { projeção VD (ventrodorsal) ou DV (dorsoventral). }\end{array}$ \\
\hline & 8. Veia cava caudal em direção dorsocranial. \\
\hline \multirow{5}{*}{$\begin{array}{l}\text { Aumento do } \\
\text { átrio esquerdo }\end{array}$} & $\begin{array}{l}\text { 1. Concavidade dorsocaudal em margem caudal da silhueta cardíaca em projeções LL } \\
\text { (laterolaterais). }\end{array}$ \\
\hline & $\begin{array}{l}\text { 2. Abaulamento da borda cardíaca esquerda entre às } 2 \text { e } 3 \text { h em projeção VD ou DV - } \\
\text { aumento da aurícula esquerda. }\end{array}$ \\
\hline & $\begin{array}{l}\text { 3. Efeito de parede dupla por aumento de radiopacidade sobreposta à silhueta cardíaca } \\
\text { em projeção VD ou DV. }\end{array}$ \\
\hline & 4. Deslocamento dorsal da traqueia terminal e tronco brônquico principal \\
\hline & $\begin{array}{l}\text { 5. Divergência dos brônquios principais em projeção VD ou DV, com separação em } \\
\text { forma de "V" ou "U". }\end{array}$ \\
\hline \multirow{5}{*}{$\begin{array}{l}\text { Aumento do } \\
\text { ventrículo } \\
\text { esquerdo }\end{array}$} & 1. Borda cardíaca caudal ereta e se estendendo mais caudalmente em projeções LL. \\
\hline & 2. Ápice cardíaco arredondado. \\
\hline & $\begin{array}{l}\text { 3. Borda cardíaca esquerda arredondada e se aproximando da parede torácica em } \\
\text { projeção VD ou DV. }\end{array}$ \\
\hline & 4. Deslocamento dorsal da traqueia intratorácica e tronco brônquico principal. \\
\hline & em margem cranial da silhuet \\
\hline Aum & LL. \\
\hline átrio & 2. Abaulamento da borda cardíaca direita entre \\
\hline & 3. Deslocamento dorsal da traqueia, cranial à c \\
\hline \multirow{5}{*}{$\begin{array}{l}\text { Aumento do } \\
\text { ventrículo } \\
\text { direito }\end{array}$} & 1. Aumento do contato cardioesternal em projeções LL. \\
\hline & 2. Deslocamento dorsal do ápice cardíaco em relação ao esterno em projeções LL. \\
\hline & 5. A \\
\hline & 4. Borda cardíaca direita abaulada e se aproximando da parede torácica em projeção \\
\hline & $\begin{array}{l}\text { VD ou DV, dando à silhueta cardí } \\
5 \text {. Deslocamento dorsal da traquei }\end{array}$ \\
\hline
\end{tabular}

As imagens do eixo-longo e do eixo-curto e as medidas ecocardiográficas foram obtidas com o animal em decúbito lateral direito, posicionandose o transdutor na janela paraesternal direita, localizada entre o terceiro e sexto espaços intercostais (EIC) direitos, entre o esterno e a articulação costocondral. A partir do eixo longo, foram obtidas as visões quatro-câmaras e trato de saída do ventrículo esquerdo. Já no eixo curto, foram observados os cortes nos níveis apical, papilar, cordal, mitral e aórtico.
As imagens ultrassonográficas foram inicialmente obtidas em modo B, avaliando-se as relações anatômicas entre as estruturas, a contratilidade e os aspectos morfofuncionais valvares. A presença de jatos de regurgitação valvar foi analisada com o Doppler colorido, e o gradiente de pressão da valva pulmonar por meio do Doppler pulsátil. Ainda no modo-B, foram realizadas as medições de átrio esquerdo (AE) e aorta (Ao), no plano dos vasos da base do eixo transversal, permitindo o cálculo da relação átrio esquerdo/aorta (AE:Ao). Essa relação indica aumento do átrio esquerdo, que é considerado 
ausente quando menor que 1,4 ; discreto, entre 1,4 e 1,6; moderado, entre 1,6 e 1,8 ; e grave, maior que 1,8 (Boon, 2011).

O eixo curto serviu de orientação para a obtenção das imagens em modo $\mathrm{M}$, com exposição simultânea do modo bidimensional na tela. As variáveis ecocardiográficas analisadas incluíram: diâmetro interno do ventrículo esquerdo na diástole e na sístole (DIVEd e DIVEs), espessura do septo interventricular na diástole (SIVd), espessura da parede livre do ventrículo esquerdo no final da diástole (PPVEd) e diâmetro do ventrículo direito na diástole (DVDId).

Essas variáveis foram calculadas com base em imagens ecocardiográficas obtidas por meio do modo $\mathrm{M}$ da janela paresternal direita, em seu eixo transversal, no plano das cordas tendíneas, sendo considerada como valor final a média obtida de três ciclos cardíacos. Com bases nessas medidas, foram calculados os índices funcionais, como a fração de encurtamento (FS) e o tempo de relaxamento isovolumétrico (IVRT), conforme descritos por Bonagura (1983).

Já na janela apical esquerda, foram analisadas as ondas E' e A', obtidas por meio do Doppler tecidual do anel mitral lateral. Pela janela paraesternal caudal esquerda (apical), em modo bidimensional, foram observadas as relações anatômicas entre as estruturas cardíacas, os aspectos morfofuncionais valvares e a contratilidade miocárdica.

Então, estatística descritiva foi utilizada para expor os achados radiográficos subjetivos sugestivos de aumento das câmaras cardíacas, segundo a classificação por escore, em comparação ao aumento verdadeiro determinado pela ecocardiografia. Com base nisso, foi possível o cálculo da sensibilidade, da especificidade, da acurácia e do índice de confiabilidade do exame radiográfico (Pereira, 1995).

Ainda, análise de correlação de Pearson (r) foi realizada para cada paciente em relação aos índices ecocardiográficos e aos achados radiográficos objetivos, incluindo VHS, eixo longo e eixo curto. Os valores de $r$ foram submetidos a teste de significância por meio do teste t, considerando-se como significativo
$\mathrm{P}<0,05$. Diagramas de dispersão foram obtidos para representação do comportamento de correlação entre as variáveis radiográficas objetivas e os índices ecocardiográficos (Ogliari e Andrade, 2005).

\section{RESULTADOS}

Foram selecionados 104 caninos para o estudo, sendo 67 fêmeas e 37 machos, com média de idade de 10,1 anos $( \pm 4,4$ anos $)$ e peso médio de $13,5 \mathrm{~kg}( \pm 4,6 \mathrm{~kg})$, incluindo diferentes raças, com destaque aos sem raça definida (30/104), seguidos por Australian Cattle Dog (17/104); Poodle Miniatura (15/104); Pinscher e Dachshund (6/104); Cocker Spaniel (4/104); York Shire, Shih Tzu, Beagle e Boxer (2/104); Lhasa Apso e Schnauzer Miniatura (1/104).

Em relação ao átrio esquerdo, enquanto $62,5 \%$ (65/104) dos pacientes não possuíam alterações radiográficas condizentes com o aumento dessa câmara, somente $75 \%$ (49/65) desses realmente não apresentavam alterações no ecocardiograma (Fig. 1), ou seja, não se observou alteração radiográfica em 16 casos em que o ecocardiograma apontou aumento. Dos 62 pacientes com relação AE:Ao abaixo de 1,4 (normal), 13 deles tiveram aumento indicado pela radiografia (Tab. 2).

Entre os 39 pacientes caracterizados radiograficamente como alterados em relação ao átrio esquerdo, somente 13 foram classificados de forma correta quanto ao grau de aumento, sendo sete com alteração discreta e seis, grave. Todos os 13 pacientes que possuíam aumento grave do átrio esquerdo no ecocardiograma foram avaliados como aumentados pela radiografia, mesmo que com classificação em graus distintos em alguns casos.

Quanto ao ventrículo esquerdo, enquanto $87,5 \%$ (91/104) não tinham alterações estruturais no ecocardiograma, somente $63,5 \%$ (66/104) foram classificados como tal por meio da radiografia (Fig. 1). Em dois animais, havia aumento da câmara não detectado pelo exame radiográfico, e em 27, o aumento foi indicado pela radiografia, quando, na realidade, não existia na ecocardiografia; entre esses 27 pacientes, um total de 16 possuía aumento somente do átrio esquerdo (Tab. 2). 


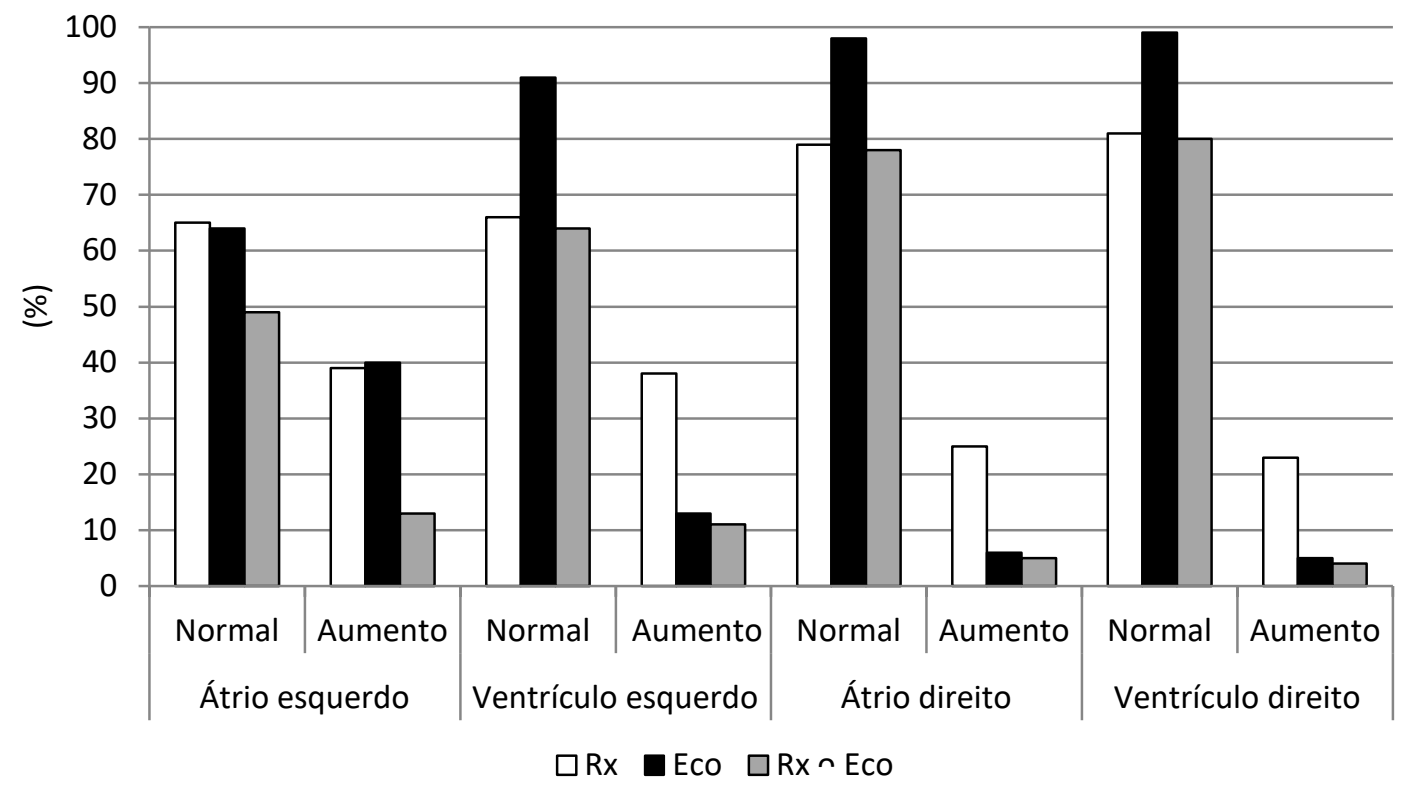

Figura 1. Quantidade relativa (\%) de caninos no presente estudo classificados como normais, com aumento do átrio esquerdo, ventrículo esquerdo, átrio direito ou ventrículo direito, conforme os exames radiográfico $(\mathrm{Rx})$, ecocardiográfico (Eco) e simultaneamente em ambos os exames $(\mathrm{Rx} \sim$ Eco).

Tabela 2. Número de caninos classificados como positivos (+) ou negativos (-) para aumento global do coração ou de câmaras cardíacas específicas, segundo a radiografia e a ecocardiografia. Sensibilidade, especificidade, acurácia e índice de confiabilidade (Kappa) do exame radiográfico em relação à detecção de aumento específico das câmaras cardíacas e aumento global determinado pelo método VHS, em comparação ao ecocardiograma

\begin{tabular}{lccccccc}
\multicolumn{1}{c}{ Avaliação } & \multicolumn{1}{c}{ Eco +} & Eco - & Sensibilidade & Especificidade & Acurácia & Kappa \\
\hline \multirow{2}{*}{ Átrio esquerdo } & $\mathrm{Rx}+$ & 26 & 13 & $65,0 \%$ & $79,0 \%$ & $72,1 \%$ & 0,43 \\
& $\mathrm{Rx}-$ & 16 & 49 & & & & \\
Ventrículo & $\mathrm{Rx}+$ & 11 & 27 & $84,6 \%$ & $70,3 \%$ & $72,1 \%$ & 0,44 \\
esquerdo & $\mathrm{Rx}-$ & 2 & 64 & & & & \\
& $\mathrm{Rx}+$ & 5 & 20 & $83,3 \%$ & $79,6 \%$ & $79,8 \%$ & 0,35 \\
Átrio direito & $\mathrm{Rx}-$ & 1 & 78 & & & & \\
& $\mathrm{Rx}+$ & 4 & 19 & $80,0 \%$ & $80,8 \%$ & $80,8 \%$ & 0,33 \\
Ventrículo & $\mathrm{Rx}-$ & 1 & 80 & & & & \\
direito & $\mathrm{Rx}+$ & 25 & 32 & $56,8 \%$ & $46,7 \%$ & $50,9 \%$ & 0,50 \\
Cardiomegalia & $\mathrm{Rx}-$ & 19 & 28 & & & & \\
(VHS) &
\end{tabular}

Eco + = aumento da câmara ou global da silhueta cardíaca por meio do ecocardiograma; Eco - = ausência de aumento por meio do ecocardiograma; $\mathrm{Rx}+=$ aumento da câmara ou global da silhueta cardíaca por meio da radiografia; Rx = ausência de aumento cardíaco por meio da radiografia.

Em relação ao aumento das câmaras cardíacas direitas, $24 \%(25 / 104)$ das radiografias demonstravam aumento em átrio direito, contudo somente 4,8\% (5/104) realmente o apresentavam
(Fig. 1), indicando a ocorrência de 20 casos falsos positivos nos exames radiográficos. Em apenas um animal (1/6) com aumento dessa câmara detectado no exame ecocardiográfico, 
esse aumento não foi demonstrado pela radiografia (Tab. 2).

Enquanto 95,2\% (99/104) dos pacientes não apresentavam alterações no ventrículo direito pelo ecocardiograma, 22,1\% (23/104) dos exames radiográficos indicavam aumento da câmara (Fig. 1), ou seja, 19 casos falsos positivos pela radiografia. Também houve um paciente que mostrava aumento da câmara, entretanto foi classificado radiograficamente como normal (Tab. 2), isto é, um falso negativo.

A sensibilidade do exame radiográfico quanto ao aumento específico das câmaras cardíacas variou entre $65 \%$ para o átrio esquerdo e $84,6 \%$ para o ventrículo esquerdo; já em relação à cardiomegalia detectada pelo VHS, houve $56,8 \%$ de sensibilidade. A especificidade do método VHS foi de 46,7\%; já para as câmaras individualmente este oscilou entre $70,3 \%$ para o ventrículo esquerdo e $80,8 \%$ para o ventrículo direito. A acurácia da radiografia na detecção de aumento global da silhueta cardíaca foi de 50,9\%; já em relação às câmaras cardíacas esquerdas e ao ventrículo direito, respectivamente, variou entre $72,1 \%$ e $80,8 \%$. O índice de confiabilidade da avaliação radiográfica não ultrapassou 0,5 (Kappa) (Tab. 2).

Com nível de significância de 0,05 (p), foi encontrada correlação positiva entre a variável radiográfica VHS e o índice ecocardiográfico Ae:Ao. A variável eixo curto correlacionou-se de forma positiva com Ae:Ao e velocidade da onda E', enquanto o eixo longo não apresentou correlação positiva entre qualquer das variáveis avaliadas. As variáveis ecocardiográficas DVDId, SIVd, DIVEd, PPVEd, DIVEs, velocidade da onda $A^{\prime}$ e tempo IVRT não apresentaram correlação significativa com as avaliações objetivas radiográficas (Tab. 3).

Os gráficos de dispersão demonstram a distribuição dos valores de VHS e eixo curto em relação aos índices ecocardiográficos, com os quais houve correlação linear significativa (Fig. 2).

Tabela 3. Valores do $r$ de Pearson e do teste de significância (p) encontrados na análise de correlação entre as variáveis objetivas ecocardiográficas (Ae:Ao, DVDId, SIVd, DIVEd, PPVEd, DIVEs, Vel. E', Vel. A', tempo IVRT) e radiográficas (VHS, eixos curto e longo) obtidas de 104 caninos estudados

\begin{tabular}{lcccccc}
\hline \multirow{2}{*}{$\begin{array}{c}\text { Variáveis } \\
\text { ecocardiográficas }\end{array}$} & \multicolumn{3}{c}{ VHS } & \multicolumn{4}{c}{ Eariáveis radiográficas } \\
\cline { 2 - 7 } & $r$ & $p$ & $r$ & $p$ & \multicolumn{2}{c}{ Eixo longo } \\
\hline Ae:Ao & 0,3136 & $0,0013^{*}$ & 0,3813 & $0,0001^{*}$ & 0,1648 & 0,0978 \\
DVDId & 0,0839 & 0,4089 & 0,0633 & 0,5337 & 0,0365 & 0,7199 \\
SIVd & 0,0146 & 0,8831 & $-0,0327$ & 0,7438 & $-0,0373$ & 0,7067 \\
DIVEd & 0,1454 & 0,1447 & 0,1389 & 0,1618 & 0,0943 & 0,3436 \\
PPVEd & 0,1450 & 0,1438 & 0,1475 & 0,1371 & 0,0801 & 0,4215 \\
DIVEs & 0,1365 & 0,1692 & 0,1383 & 0,1613 & 0,1009 & 0,3104 \\
Vel. E' & 0,1907 & 0,0525 & 0,2021 & $0,0397 *$ & 0,1245 & 0,2080 \\
Vel. A' & 0,0004 & 0,9969 & 0,0159 & 0,8723 & $-0,0082$ & 0,9341 \\
Tempo IVRT & $-0,1450$ & 0,1440 & $-0,1508$ & 0,1284 & $-0,0786$ & 0,4299 \\
\hline
\end{tabular}

* Correlação significativa pelo teste t, considerando $\mathrm{P}<0,05$. Ae:Ao = relação átrio esquerdo/aorta; DVDId = diâmetro do ventrículo direito na diástole; SIVd = espessura do septo interventricular na diástole; DIVEd e DIVEs = diâmetro interno do ventrículo esquerdo na diástole e na sístole, respectivamente; PPVEd = espessura da parede livre do ventrículo esquerdo no final da diástole; Vel. E' e Vel. A' = velocidade das ondas E' e A', respectivamente; tempo IVRT = tempo de relaxamento isovolumétrico; VHS = vertebral heart size; eixos curto $\mathrm{e}$ longo = eixos perpendiculares (maior e menor) da silhueta cardíaca. 

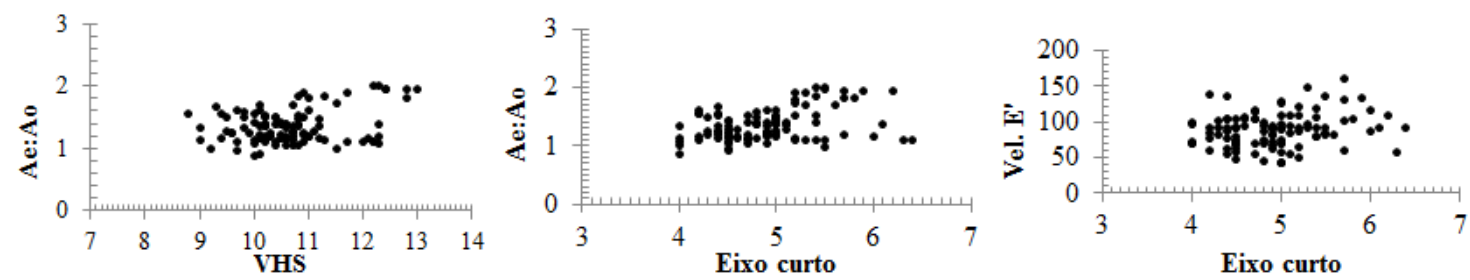

Figura 2. Gráficos de dispersão demonstrando a distribuição dos valores do VHS e eixo curto da silhueta cardíaca por meio da radiografia, em relação aos índices ecocardiográficos com os quais se correlacionaram (Ae:Ao e velocidade da onda E), nos caninos estudados.

\section{DISCUSSÃO}

Este estudo teve por objetivo determinar a relação existente entre as variáveis radiográficas objetivas e os índices ecocardiográficos, durante avaliação da cardiomegalia. Foi possível, ainda, investigar a acurácia da avaliação radiográfica subjetiva em relação à forma e ao tamanho do coração.

No que se refere à avaliação radiográfica subjetiva da silhueta cardíaca, percebe-se acurácia moderada da radiografia na detecção de aumento verdadeiro das câmaras, variando entre $72,1 \%$ na avaliação das câmaras esquerdas até $80,8 \%$ na análise do ventrículo direito. Com variação da sensibilidade entre $65 \%$ e $84,6 \%$ e especificidade oscilando entre 70,35 e 80,8\%, apreende-se que a radiografia não estima, de maneira precisa e exata, as variações morfológicas da silhueta cardíaca.

A maior sensibilidade foi encontrada na avaliação do ventrículo esquerdo, contudo se nota que a mesma câmara deteve a menor especificidade no estudo. Em 27 cães foi indicado aumento de ventrículo esquerdo pela radiografia, quando, na verdade, esse aumento não existia na ecocardiografia (Tab. 2); em 16 desses casos, havia aumento do átrio esquerdo. Dessa forma, suspeita-se que a menor especificidade esteja relacionada à influência da avaliação da gravidade da alteração ventricular em relação à alteração atrial das câmaras esquerdas, com a tendência de superestimar as alterações ventriculares em razão de aumento atrial (Lombard e Spencer, 1985).

No que se refere às câmaras cardíacas direitas, percebe-se alta proporção de exames falsos positivos - 19,2\% na avaliação atrial e 18,3\% na análise ventricular -, contudo baixa proporção de falsos negativos - cerca de $1 \%$ em ambas -, tendo a radiografia sensibilidade e especificidade razoáveis em se tratando do aumento de tais câmaras. Supõe-se que o exame radiográfico superestime as dimensões das câmaras cardíacas direitas, principalmente devido à maior variação quanto à normalidade, levando em consideração o acúmulo de gordura pericárdica, como já descrito em gatos (Litster e Buchanan, 2000), e as diferentes conformações torácicas entre as diversas raças de cães (Lamb et al., 2001).

Entre 22 casos falsos positivos em relação ao aumento de átrio e ventrículo direitos, 14 eram cães de raças condrodistróficas ou com tendência condrodistrófica, os quais apresentam tórax largo e pouco profundo, incluindo Dachshund, Shih Tzu, Lhasa Apso, Poodle e Cocker. Há uma tendência em superestimar o tamanho de tais câmaras devido ao aspecto mais globoso da silhueta cardíaca nesses pacientes, além do aparente aumento do contato cardioesternal mimetizando a cardiomegalia direita (Lamb et al., 2001; Johnson et al., 2008).

Em dois animais nos quais o átrio direito foi dado como aumentado, detectou-se no ecocardiograma uma massa em base cardíaca. A ocorrência de elevação focal da porção terminal da traqueia em estudo lateral, associada a opacidades de massa obliterando a região dorsocranial da silhueta cardíaca, em região de átrio direito, constitui o principal achado radiográfico relacionado a massas de base cardíaca (Guglielmini et al., 2016), o que pode causar dúvidas e falsos positivos para aumento atrial. Em estudo prévio, a sensibilidade da radiografia torácica na detecção de massas em base cardíaca mostrou-se baixa, apesar da alta especificidade (Guglielmini et al., 2016).

A baixa acurácia do método VHS em detectar cardiomegalia verdadeira $(50,8 \%)$ corrobora estudos anteriores, os quais demonstraram que a 
mensuração objetiva da silhueta cardíaca não adiciona valor ao diagnóstico radiográfico de cardiopatia em relação à avaliação subjetiva (Lamb et al., 2001). Nota-se significativa sobreposição dos valores do VHS entre cães normais e cães com doença cardíaca, mesmo com a utilização de valores específicos para as raças, sendo essa sobreposição possivelmente explicada pela ausência de alterações de tamanho cardíaco em pacientes com hipertrofia concêntrica ou em fase inicial da doença (Lamb e Boswood, 2002).

Em 60 pacientes, o diagnóstico ecocardiográfico foi de doença valvar mitral crônica (DVMC), sendo 36 deles com repercussão hemodinâmica, caracterizada por aumento do átrio esquerdo (relação Ae:Ao>1,4), entre os quais 28 foram corretamente avaliados de forma subjetiva pela radiografia como aumentados. Isso vai ao encontro do pressuposto de que cardiopatias que imponham sobrecarga de volume ao coração, como a DVMC, tendem a gerar aumento externo das dimensões cardíacas, possibilitando a ocorrência de sinais radiográficos associados à hipertrofia ou à dilatação da câmara (Lamb e Boswood, 2002). Entretanto, a ausência de achados radiográficos em oito pacientes com aumento verdadeiro da câmara indica que a radiografia torácica não é um exame acurado e sensível o suficiente para ser utilizado como único método de controle da doença em cães.

Ainda, entre os 36 pacientes diagnosticados com DVMC com repercussão hemodinâmica, 22 deles não apresentaram aumento do VHS na radiografia. Apesar de a cardiomegalia ter sido um achado comum em estudo realizado por Soares et al. (2005) em pacientes com DVMC e regurgitação mitral moderada a grave, no presente estudo o método radiográfico objetivo de mensuração cardíaca (VHS) demonstrou sensibilidade e acurácia baixas na avaliação desses animais.

O $\mathrm{r}$ de Pearson nas variáveis correlacionadas variou entre 0,2021 e 0,3813 (Tab. 3), demonstrando relação fracamente positiva, apesar de estatisticamente significativa. Isso indica uma tendência, ainda que discreta, de ocorrer alteração das variáveis radiográficas objetivas quando há alteração morfofuncional cardíaca, evidenciada de forma acurada pelo ecocardiograma.
Somente os índices ecocardiográficos ligados ao átrio esquerdo apresentaram correlação significativa, sendo a relação Ae:Ao a variável relacionada ao tamanho da câmara e à velocidade da onda E obtida a partir do fluxo transmitral. Ainda, nenhuma mensuração ecocardiográfica relacionada às câmaras direitas apresentou correlação com os achados radiográficos. Tais resultados possivelmente se devem à menor acurácia do VHS em detectar cardiomegalia verdadeira, com tendência a superestimar alterações do coração direito.

Os coeficientes de correlação encontrados diferem dos resultados obtidos por Nakayama $e t$ al. (2001), único estudo encontrado pelos autores que utilizou estatística semelhante, o qual indicou altos índices de correlação $(r>0,7)$ entre variáveis radiográficas, ecocardiográficas e eletrocardiográficas. Contudo, cabe ressaltar que, em tal estudo, foram utilizados 16 cães de grande porte considerados saudáveis, nos quais a cardiomegalia fora induzida por meio de um eletrodo bipolar posicionado no ventrículo direito e um marcapasso, permitindo que as variáveis fossem avaliadas progressivamente, de acordo com graus conhecidos de aumento cardíaco. Além disso, o marcapasso induziu dilatação do coração, permitindo aumento biatrial e biventricular em proporções semelhantes, o que é incomum nas doenças comumente acompanhadas na rotina, como nos pacientes avaliados no presente estudo.

\section{CONCLUSÃO}

Por fim, é possível inferir que as variáveis radiográficas objetivas de mensuração cardíaca, incluindo VHS, eixo longo e eixo curto, apresentam correlação fraca ou inexistente com os índices ecocardiográficos indicativos de aumento cardíaco, sendo o VHS um método de baixa acurácia, sensibilidade e especificidade no diagnóstico de cardiomegalia. Ainda, apesar de a avaliação radiográfica subjetiva da silhueta cardíaca demonstrar acurácia razoável na detecção de aumento das câmaras, o exame radiográfico não apresenta sensibilidade e especificidade suficiente para ser utilizado como método único e independente no diagnóstico e acompanhamento de caninos cardiopatas. A fraca correlação detectada entre os achados radiográficos objetivos e os índices ecocardiográficos indicativos de aumento 
cardíaco sugere que o estudo radiográfico não pode ser utilizado isoladamente na avaliação da cardiomegalia.

\section{REFERÊNCIAS}

ARMSTRONG, W.F.; FEIGENBAUM, H.; RYAN, T. Ecocardiografia. 7.ed. Rio de Janeiro: Guanabara Koogan, 2005. 796p.

BONAGURA, J.D. M-Mode echocardiography. Vet. Clin. N. Am. Small Anim. Pract., v.13, p.299-319, 1983.

BOON, J.A. Veterinary echocardiography. 2.ed. Oxford: Wiley-Blackwell, 2011. 480p.

BUCHANAN, J.W.; BÜCHELER, J. Vertebral scale system to measure canine heart size in radiographs. $J$. Am. Vet. Med. Assoc., v.206, p.194-199, 1995.

FERASIN, L.; CREWS, L.; BILLER, D.S. et al. Risk factors for coughing in dogs with naturally acquired myxomatous mitral valve disease. J. Vet. Intern. Med., v.27, p.286-292, 2013.

GUGLIELMINI, C.; TOALDO, M.B.; QUINCI, C. et al. Sensitivity, specificity, and interobserver variability of survey thoracic radiography for the detection of heart base masses in dogs. J. Am. Vet. Med. Assoc., v.248, p.1391-1398, 2016.

HANSSON, K.; HÄGGSTRÖM, J.; KVART, C.; LORD, P. Reader performance in radiographic diagnosis of signs of mitral regurgitation in cavalier King Charles spaniels. J. Small Anim. Pract., v.50, p.44-53, 2009.

JEPSEN-GRANT, K.; POLLARD, R.E.; JOHNSON, L.R. Vertebral heart scores in eight dog breeds. Vet. Radiol. Ultrasound, v.54, p.3-8, 2013.

JOHNSON, V.; HANSSON, K.; MAI, W. et al. The heart and major vessels. In: SCHWARTZ, T.; JOHNSON, V. (Eds.). BSAVA manual of canine and feline thoracic imaging. Quedgeley: British Small Animal Veterinary Association, 2008. p.86-176.

KIENLE, R.D.; THOMAS, W.P. Ecocardiografia. In: NYLAND, T.G.; MATTOON, J.S. Ultrassom diagnóstico em pequenos animais. 2.ed. São Paulo: Roca, 2005. p.365-438.

LAMB, C.R.; BOSWOOD, A. Role of survey radiography in diagnosing canine cardiac disease. Comp. Contin. Educ. Vet., v.24, p.316-327, 2002.
LAMB, C.R.; WIKELEY, H.; BOSWOOD, A.; PFEIFFER, D.U. Use of breed-specific ranges for the vertebral heart scale as an aid to the radiographic diagnosis of cardiac disease in dogs. Vet. Rec., v.18, p.707-711, 2001.

LITSTER, A.L.; BUCHANAN, J.W. Radiographic and echocardiographic measurement of the heart in obese cats. Vet. Radiol. Ultrasound, v.41, p.320-325, 2000.

LOMBARD, C.W.; SPENCER, C.P. Correlation of radiographic, echocardiographic and electrocardiographic signs of left heart enlargement in dogs with mitral regurgitation. Vet. Radiol., v.26, p.89-97, 1985.

NAKAYAMA, H.; NAKAYAMA, T.; HAMLIN, R.L. Correlation of cardiac enlargement as assessed by vertebral heart size and echocardiographic and electrocardiographic findings in dogs with evolving cardiomegaly due to rapid ventricular pacing. J. Vet. Intern. Med., v.15, p.217-221, 2001.

OGLIARI, P.J.; ANDRADE, D.F. Estatística básica para as ciências agronômicas e biológicas: com noções de experimentação. Florianópolis: UFSC, 2005. 358p.

OLIVE, J.; JAVARD, R.; SPECCHI, S. et al. Effect of cardiac and respiratory cycles on vertebral heart score measured on fluoroscopic images of healthy dogs. $J$. Am. Vet. Assoc., v.246, p.1091-1097, 2015.

PEREIRA, M.G. Epidemiologia: teoria e prática. Rio de Janeiro: Guanabara Koogan, 1995. 583p.

PINTO, A.C.; IWASAKI, M. Radiographic evaluation of the cardiac silhouette in clinically normal Poodles through the vertebral heart size (VHS) method. Braz. J. Vet. Res. Anim. Sci., v.41, p.261-267, 2004.

RUEHL, W.W.; THRALL, D.E. The effect of dorsal versus ventral recumbency on the radiographic appearance of the canine thorax. Vet. Radiol., v.22, p.10-16, 1981

SOARES, E.C.; LARSSON, M.H.M.A.; YAMATO, R.J. Chronic valvular disease: correlation between clinical, electrocardiographic, radiographic and echocardiographic aspects in dogs. Arq. Bras. Med. Vet. Zootec., v.57, p.436-441, 2005.

THOMAS, W.P.; GABER, C.E.; JACOBS, G.J. et al. Recommendations for standards in transthoracic twodimensional echocardiography in the dog and cat. $J$. Vet. Intern. Med., v.7, p.247-252, 1993. 